\title{
Effects of changing mosquito host searching behaviour on the cost effectiveness of a mass distribution of long-lasting, insecticidal nets: a modelling study
}

Olivier JT Briët ${ }^{1,2^{*}}$ and Nakul Chitnis ${ }^{1,2}$

\begin{abstract}
Background: The effectiveness of long-lasting, insecticidal nets (LLINs) in preventing malaria is threatened by the changing biting behaviour of mosquitoes, from nocturnal and endophagic to crepuscular and exophagic, and by their increasing resistance to insecticides.

Methods: Using epidemiological stochastic simulation models, we studied the impact of a mass LLIN distribution on Plasmodium falciparum malaria. Specifically, we looked at impact in terms of episodes prevented during the effective life of the batch and in terms of net health benefits (NHB) expressed in disability adjusted life years (DALYs) averted, depending on biting behaviour, resistance (as measured in experimental hut studies), and on pre-intervention transmission levels.
\end{abstract}

Results: Results were very sensitive to assumptions about the probabilistic nature of host searching behaviour. With a shift towards crepuscular biting, under the assumption that individual mosquitoes repeat their behaviour each gonotrophic cycle, LLIN effectiveness was far less than when individual mosquitoes were assumed to vary their behaviour between gonotrophic cycles. LLIN effectiveness was equally sensitive to variations in host-searching behaviour (if repeated) and to variations in resistance. LLIN effectiveness was most sensitive to pre-intervention transmission level, with LLINs being least effective at both very low and very high transmission levels, and most effective at around four infectious bites per adult per year. A single LLIN distribution round remained cost effective, except in transmission settings with a pre-intervention inoculation rate of over 128 bites per year and with resistant mosquitoes that displayed a high proportion (over 40\%) of determined crepuscular host searching, where some model variants showed negative NHB.

Conclusions: Shifts towards crepuscular host searching behaviour can be as important in reducing LLIN effectiveness and cost effectiveness as resistance to pyrethroids. As resistance to insecticides is likely to slow down the development of behavioural resistance and vice versa, the two types of resistance are unlikely to occur within the same mosquito population. LLINs are likely cost effective interventions against malaria, even in areas with strong resistance to pyrethroids or where a large proportion of host-mosquito contact occurs during times when LLIN users are not under their nets.

Keywords: Behavioural resistance, Physiological resistance, LLINs, Open malaria, ITNs, Mosquito, Modelling, Insecticide

\footnotetext{
* Correspondence: olivier.briet@unibas.ch

'Department of Epidemiology and Public Health, Swiss Tropical and Public

Health Institute, Basel, Switzerland

2University of Basel, Basel, Switzerland
}

\section{Biomed Central}

(c) 2013 Briët and Chitnis; licensee BioMed Central Ltd. This is an Open Access article distributed under the terms of the Creative Commons Attribution License (http://creativecommons.org/licenses/by/2.0), which permits unrestricted use, distribution, and reproduction in any medium, provided the original work is properly cited. 


\section{Background}

Long-lasting insecticidal net (LLIN) distribution campaigns have played an important role in recent successes in malaria control [1]. LLINs protect against mosquito bites that mostly occur indoors during sleeping hours, coinciding with times when people are using LLINs. Furthermore, the nets have a protective 'community effect' by killing mosquitoes [2], thereby reducing the probability that the infected mosquito will survive the extrinsic incubation period and become infectious.

Resistance to pyrethroids in mosquito populations is increasing [3], threatening the effectiveness of pyrethroidbased interventions such as LLINs [4]. LLIN effectiveness is also threatened by shifts in biting behaviour, from nocturnal towards crepuscular, with mosquitoes actively host searching during the early morning and/or evening, when many LLIN users are not under their nets [5-7]. For example, Russell and colleagues [8] observed increased outdoor feeding after exposure to insecticide treated nets (ITNs) in Tanzania. Such a shift could occur simply ifmosquitoes are unsuccessful in finding a blood meal during their normal active host-searching period and if mosquitoes learn $[9,10]$ and repeat behaviour that resulted in a blood meal The above causes of these shifts can be described as phenotypic plasticity [5]. It could occur if mosquitoes that search for hosts indoors during sleeping hours have a higher risk of being killed (by LLINs). If there is a genetic basis for the behaviour [11], pressure from LLINs could select for alleles that are associated with crepuscular biting behaviour, resulting in decreased exposure of mosquitoes to LLINs, and decreased effectiveness of LLINs over time. If these alleles are associated with certain (sub) species, this could lead to differential suppression of (sub) species $[8,12]$.

In this paper, 'behavioural resistance' is defined as the behaviour-related ability to be unaffected by something. Such ability can, but does not need to be, acquired (or evolved) in response to pressure. Mosquitoes that tend to bite during times that LLIN users are not under their nets are less affected by LLINs than nocturnal host-searching mosquitoes, and thus have some 'behavioural resistance' against LLINs. However, mosquitoes that avoid contact with LLINs are not necessarily more behaviourally resistant than mosquitoes that do not if the contact-avoiding mosquitoes subsequently fail to find a blood meal or have a lower survival rate associated with crepuscular activity.

If host-searching behaviour varies in the vector population, this has implications for LLIN effectiveness. Molineaux and colleagues [13] show that with the assumption of non-uniform exposure to insecticides, vector control tools are much less effective than under the usual implicit assumption of uniform exposure. If all female mosquitoes in a population have the same stochastic probability of behaving a certain way each gonotrophic cycle, then mosquito potential exposure to LLINs is uniform. If, at the other extreme, each female always repeats her behaviour each gonotrophic cycle, her behaviour being determined before (genetically or phenotypically) or during the first gonotrophic cycle (through learning from experience), behaviour is fully determined and potential exposure is non-uniform. In the former fully 'probabilistic' case, each mosquito has a priori the same chance of surviving each gonotrophic cycle. In the latter, fully 'determined' case, those mosquitoes that never search for human hosts indoors during human sleeping hours are never exposed to LLINs, and thus their survival is not affected by LLINs. While behaviour is unlikely to be fully probabilistic, it is also unlikely to be fully determined. In reality, the degree of determinedness will lie somewhere in between these two extremes.

The proportion of mosquito-host encounters that occur indoors during sleeping hours in the absence of bed nets gives an indication of how strongly the mosquito population can be affected by LLINs. The more crepuscular and exophagic the vector population, the lower this proportion. This proportion, also called the ' $\pi_{i}$ value' [14] or ' $\pi_{s}$ value' [15], can also be defined as "...the proportion of normal exposure of unprotected humans lacking nets that occurs at times and places when net users would be protected by sleeping under them" [16]. If host-searching behaviour is fully determined, LLINs cannot reduce exposure to bites beyond the level that occurred during human sleeping hours prior to invention. In such a case, and if mosquitoes are killed by LLINs, the $\pi_{i}$ value will be lower during intervention than prior to intervention. However, if there is some stochasticity in the behaviour, LLINs can potentially reduce exposure beyond the level occurring during human sleeping hours prior to intervention, because all mosquitoes are at risk of being killed by LLINs.

A previous modelling study [17] quantified the sensitivity of the effectiveness of a mass distributed LLIN batch to insecticide resistance, as measured in experimental hut studies ${ }^{\mathrm{a}}$. In that study, the pre-intervention $\pi_{\mathrm{i}}$ was kept constant at a value of 0.75 , and host-searching behaviour was assumed to be fully determined. This paper extends that previous study by incorporating sensitivity to assumptions about host-searching behaviour into the analysis.

\section{Methods}

The model used the same 'central scenario' as described earlier [17], with LLIN effect parameterization based on results from an experimental hut study with PermaNet 2.0 (P2) LLINs in Pitoa [18], where the Anopheles gambiae sensu lato population, consisting of 95\% Anopheles arabiensis and 5\% Anopheles gambiae sensu stricto, shows $70 \%$ mortality in $0.05 \%$ deltamethrin World Health 
Organization (WHO) insecticide susceptibility tests. Figure 1 illustrates the course of a central scenario simulation with a pre-intervention entomological inoculation rate (EIR) of 16 infectious bites per adult per annum (IBPAPA). In this scenario, LLINs are distributed to $70 \%$ of the simulated population in a single mass distribution in year five, after which they diminish in number following an attrition curve with a half-life of four years, and decay chemically (lose insecticide) and physically (form holes in their fabric), as shown in Figure 1a.

In this experiment, the pre- intervention $\pi_{i}$ value varied between 0.6 (representing behaviourally-resistant mosquitoes) and 0.9 (representing behaviourally-susceptible mosquitoes), with 0.75 as the central value. The degree of determinedness in host-searching behaviour in the population was examined for the two extremes: fully probabilistic (each gonotrophic cycle, a mosquito may display a host-searching behaviour different from that of the previous gonotrophic cycle), and fully determined behaviour (a mosquito displays the same behaviour each gonotrophic cycle). The latter was modelled using two separate subpopulations, one of which was potentially affected by LLINs. The transmission intensity was varied over a wide range. Also, P2 LLIN effect parameterizations [17] were included in the experiment, for mosquito populations with a varying degree of physiological resistance to deltamethrin [18-22]. Under the assumption of fully determined host-searching behaviour, only the nocturnal adult
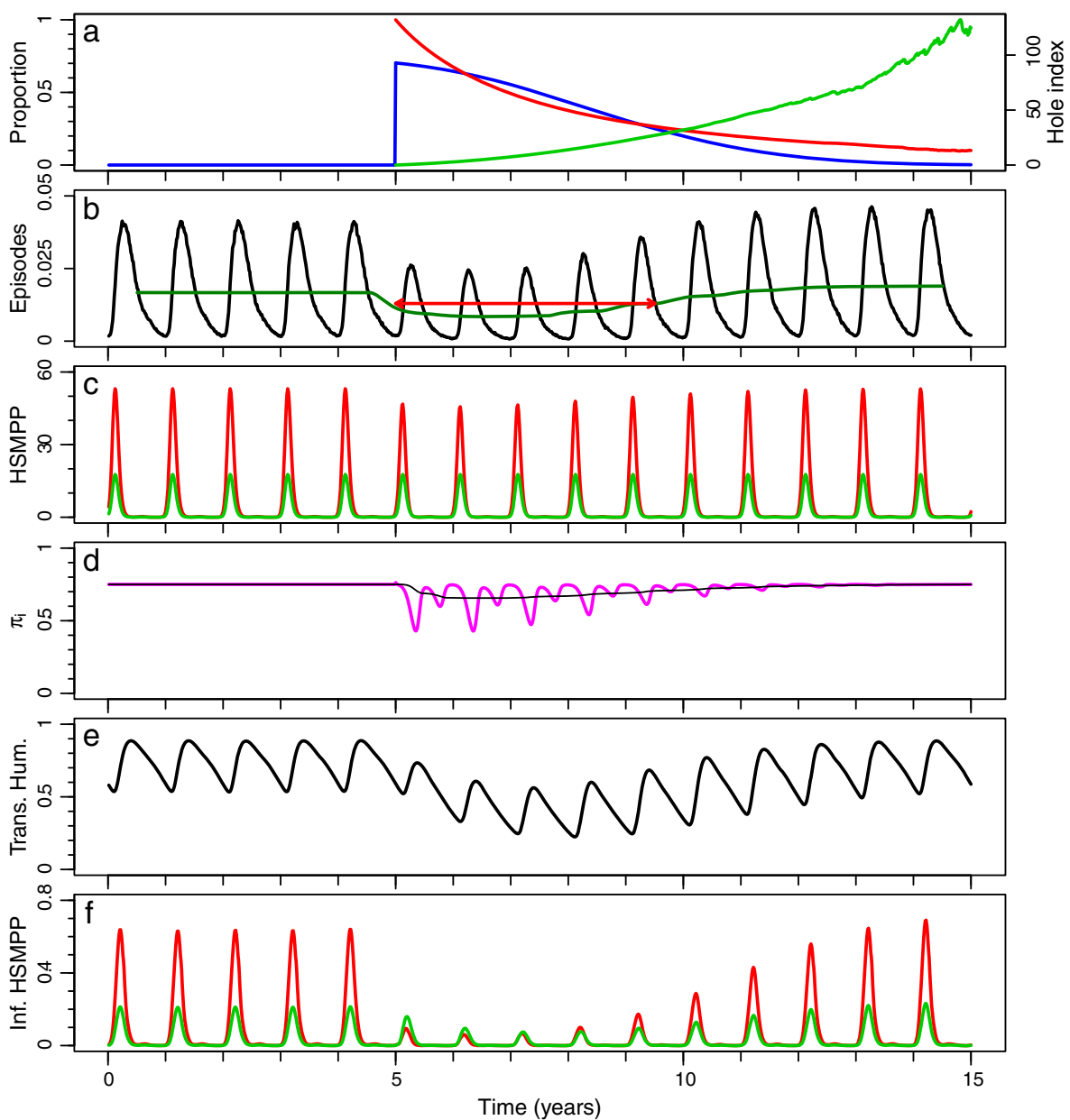

Figure 1 Central scenario simulation with base model. a) The blue line (on left vertical axis) represents the proportion of the population covered and the red line (on left vertical axis) represents the mean insecticide in the remaining LLINs as a proportion of its initial value. The light green line (on the right vertical axis) represents the mean hole index in the remaining LLINs [29]. b) The black line represents the number of episodes per person per five-day period, and the dark green line represents their one-year moving average. The red arrow indicates the approximate length of the effective lifetime of the LLIN distribution. c) Host-searching mosquitoes per person (HSMPP). The red line represents nocturnal mosquitoes and the lime green line represents crepuscular mosquitoes. d) The proportion of nocturnal mosquitoes out of the total host searching mosquitoes $\left(\pi_{i}\right)$ is represented by the magenta line. The thin black line represents the 365 day moving average. e) Proportion of humans transmitting malaria to mosquitoes. $\mathbf{f}$ ) Infectious HSMPP. The red line represents nocturnal mosquitoes and the lime green line represents crepuscular mosquitoes. 
host-searching population is directly affected by the LLINs (Figure 1c). In this scenario, host-searching behaviour is assumed to be non-genetically determined and mosquito emergence is assumed to be independent of the adult population size, thus the proportion of nocturnal mosquitoes (out of the total host-searching mosquitoes $\left.\left(\pi_{\mathrm{i}}\right)\right)$ is reduced temporarily and fluctuates strongly with the seasonal population dynamics driven mainly by seasonal fluctuations in breeding site capacity (Figure 1d). The amount of reduction in $\pi_{i}$ depends not only on the coverage and quality of the LLINs, but also on the preintervention $\pi_{\mathrm{i}}$ itself and on the susceptibility of the mosquito population (Figure 2). Note that under an assumption of fully probabilistic behaviour, the $\pi_{i}$ value during intervention remains equal to the value prior to intervention. The proportion of humans transmitting malaria to mosquitoes is also temporarily reduced (Figure 1e). The number of nocturnal infectious host-searching mosquitoes is strongly reduced by the LLINs, mostly as a result of the LLINs' impact on their survival, reducing their longevity to less than the extrinsic incubation period (Figure 1f). The number of infectious crepuscular mosquitoes is also reduced, but to a lesser extent. This is exclusively due to the reduced transmission from humans to mosquitoes. During the first three years after LLIN

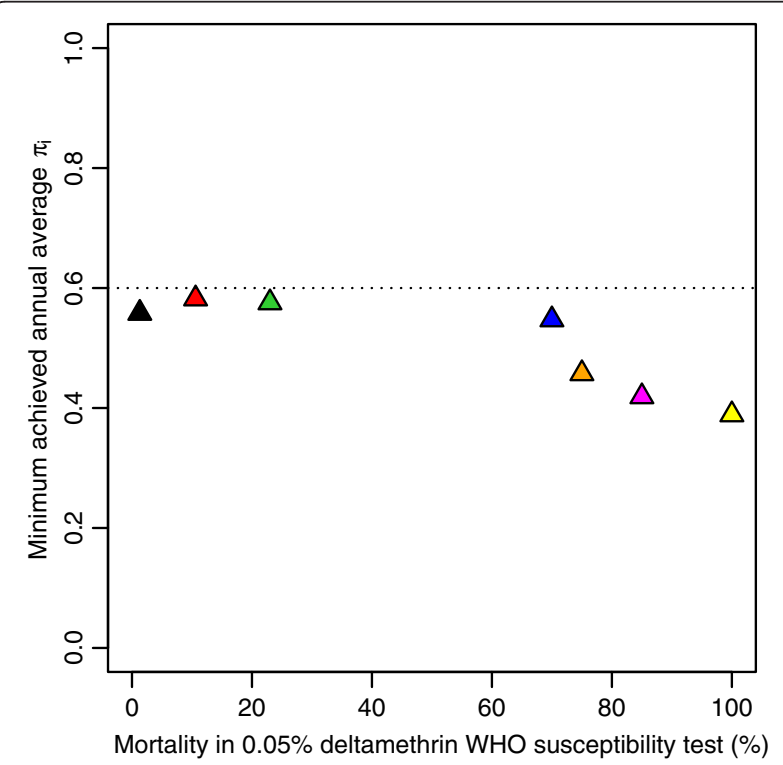

Figure 2 Minimum achieved annual average $\pi_{\mathbf{i}}$ during LLIN intervention. Minimum achieved 365-day moving average $\pi_{\mathrm{i}}$ in simulations during intervention (cf. Figure 1d) with P2 mosquito nets assuming a pre-intervention $\pi_{i}$ of 0.6 and fully determined host-searching behaviour, depending on susceptibility of the mosquito population, with black: 'Akron' [19], red: 'Yaokoffikro' [20], lime green: 'Kou' [18], dark blue: 'Pitoa' [18], orange: 'Van Duc A' [21], magenta: 'Malanville' [18], and yellow: 'Zeneti' [22]. The dotted horizontal line at $\pi_{\mathrm{i}}=0.6$ indicates the level of the pre-intervention indoor exposure when people would be protected by LLINs. distribution, transmission from crepuscular mosquitoes is more important than that from nocturnal mosquitoes.

For each scenario, ten simulations were run, each with a unique random seed, for each of 14 model variants [23] Simulations were run using the OpenMalaria modelling platform [23-25], which combines stochastic individualbased models for Plasmodium falciparum malaria in humans with a deterministic model for malaria in mosquitoes [25,26]. As was done previously [17], from each scenario output and based on the number of uncomplicated episodes, severe episodes, sequelae and deaths depending on age, disability adjusted life years (DALYs) and health system costs were calculated for each whole year during the simulation run. For intervention scenarios, the period of the epidemiological effect was determined. This was defined as the period from distribution of a batch of LLINs until the time that the effect of LLINs had waned to just over half of the maximum impact in terms of uncomplicated and severe episodes averted. This period, called the 'effective lifetime' of the batch of LLINs, is illustrated by the red arrow in Figure 1b. By comparing each scenario to its corresponding non-intervention scenario, the effectiveness of a mass distributed batch of LLINs was calculated in terms of episodes averted during the effective lifetime, and cost effectiveness in terms of net health benefits (NHB) [27], expressed in DALYs, depending on biting behaviour and pre-intervention EIR. The costs and cost savings to the health system are included in the $\mathrm{NHB}$, using a ceiling ratio conversion factor of 235.28 United States $\${ }^{2012}$ per DALY. The method for calculating DALYs and NHB is described in detail by Briët and colleagues [17] in an additional file.

\section{Results}

\section{LLIN effectiveness depending on host-searching} behaviour and transmission level

The shape of the relationship between episodes averted (Figure 3) or NHB (Figure 4) and pre-intervention EIR appears to be similar for the four combinations of assumptions about host-searching behaviour, although with fully determined behaviour and a low $\pi_{i}$ value of 0.6 , LLINs are clearly less effective.

Figures 5 and 6 show the per cent differences of episodes averted and NHB, respectively, among the four combinations of assumptions about host-searching behaviour, depending on the pre-intervention EIR. The per cent difference increased non-linearly with increasing pre-intervention EIR. Figures S1 and S2 show absolute differences [see Additional file 1: Figures S1-S2], which are the proportional differences multiplied by the magnitude of the effect in the comparator.

When the behaviour was assumed to be fully probabilistic (Figures $5 \mathrm{a}$ and 6a), the difference between 90 or $60 \%$ of the mosquito-host contact occurring during times when 


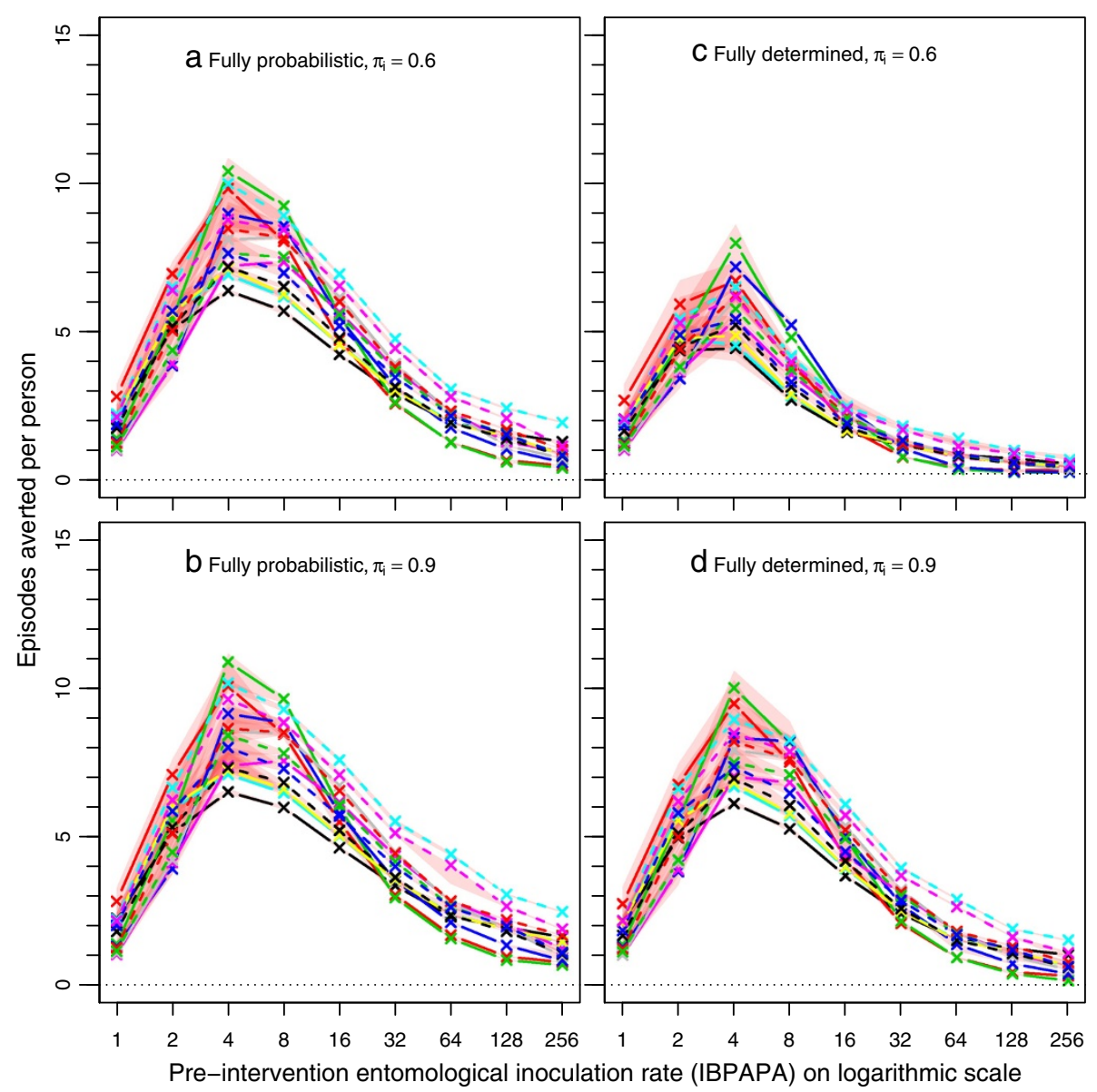

Figure 3 Episodes averted by a mass LLIN distribution depending on host-searching behaviour. Each line represents the median number of episodes averted per person of ten simulation runs (each with unique random seed) during the effective lifetime of a mass distribution, as compared to matching non-intervention scenarios, with population size $=100,000$. The red semi-transparent polygons represent the range of the ten runs. Per panel, there are 14 lines (and 14 red polygons), each representing a malaria model variant. Model variants [28]: R0000 = solid black lines; R0063 = solid red lines; R0065 = solid green lines; R0068 = solid blue lines; R0111 = solid light blue lines; R0115 = solid magenta lines; R0121 = solid yellow lines; R0125 = solid grey lines; R0131 = dashed black lines; R0132 = dashed red lines; R0133 = dashed green lines; R0670 = dashed blue lines; R0674 = dashed light blue lines; R0678 = dashed magenta lines. a) Fully probabilistic host-searching behaviour, with a $\pi_{i}$ value of 0.6 . b) Fully probabilistic host-searching behaviour, with a $\pi_{i}$ value of 0.9. c) Fully determined behaviour with a $\pi_{i}$ value of 0.6 . d) Fully determined behaviour with a $\pi_{i}$ value of 0.9 .

LLIN users would not be under their nets $\left(\pi_{i}\right.$ values of 0.9 and 0.6 , respectively) was much less pronounced (with a difference of up to about 30\%) than when the behaviour was fully determined (with a difference up to about 70\%) (Figures $5 \mathrm{~b}$ and $6 \mathrm{~b}$ ). A comparison between the assumptions of fully probabilistic and fully determined behaviour at constant $\pi_{i}$ value shows that at a $\pi_{i}$ value of 0.6 , the LLINs are much less effective with determined behaviour than with probabilistic behaviour (Figure $5 \mathrm{c}$ and Figure $6 \mathrm{c}$ ). As expected, with a high $\pi_{i}$ value of 0.9 , this difference was much less pronounced (Figures $5 \mathrm{~d}$ and $6 \mathrm{~d}$ ).

\section{Sensitivity analysis}

Figure 7 illustrates the sensitivity of the effectiveness of a mass LLIN distribution to assumptions about host-searching behaviour around a central scenario and allows comparison with results from a previous analysis on sensitivity to insecticide susceptibility and pre-intervention EIR [17]. At a pre-intervention EIR of 16 IBPAPA, the proportion of mosquito-host interaction that, prior to intervention, occurred during times when people were indoors and asleep (Panels a \& b) appears to be as important as insecticide resistance status (Panels e \& f) if host-searching behaviour was assumed to be fully determined. As noted before [29], the pre-intervention EIR was extremely important. Even if varied over a small part of its potential range, the pre-intervention EIR had more impact on the effectiveness of an LLIN distribution than the $\pi_{\mathrm{i}}$ value and insecticide resistance of mosquito populations. Panels c \& d show that (at a pre-intervention $\pi_{i}$ value of 0.75 ) the effectiveness of a 


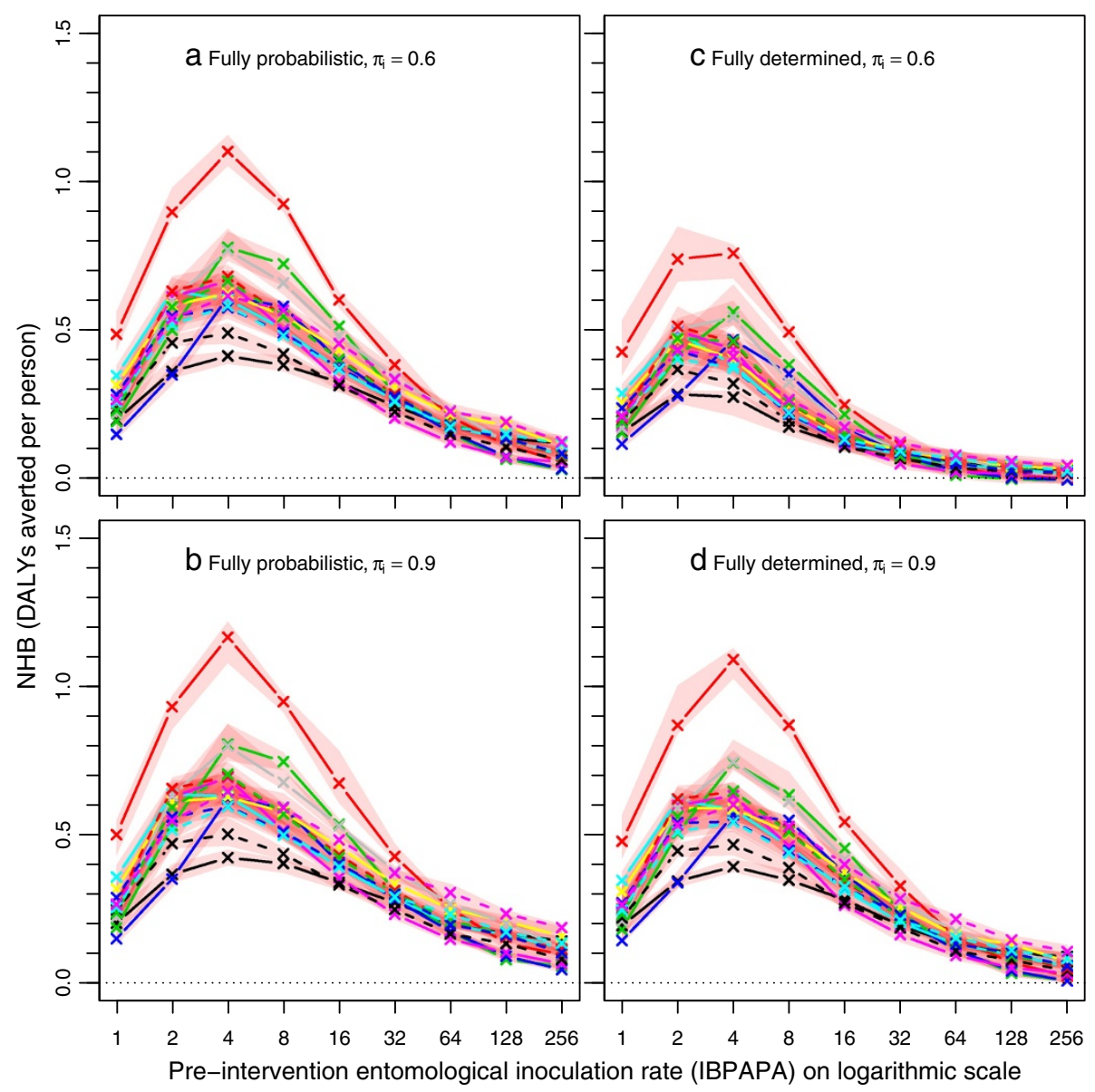

Figure 4 Net health benefits of a mass LLIN distribution, depending on host searching behaviour. Each line represents the median number of $\mathrm{NHB}$, which are expressed in DALYs averted per person of ten simulation runs (each with unique random seed) during the effective lifetime of a mass distribution, as compared to matching non-intervention scenarios, with population size $=100,000$. Legend further as in Figure 3.

mass LLIN distribution is very sensitive to the assumptions about the degree of determinedness of the host-searching behaviour: If host-searching behaviour is fully probabilistic, the LLIN effectiveness is much larger. It should be noted that under the assumption of fully probabilistic behaviour, the effectiveness is much less sensitive to variations in the $\pi_{i}$ value (see Figures $5 a$ and $6 a$ ).

\section{Discussion}

In this sensitivity analysis, a minimum pre-intervention $\pi_{i}$ value of 0.6 was used. In the literature, $\pi_{i}$ values for malaria vectors as low as 0.45 have been observed $[8,16,30]$, but such low values were in areas already having high coverage of bed nets [30,31], and were likely low as a result of LLIN use or other ITNs [8]. Under the assumption of fully probabilistic host-searching behaviour, all mosquitoes have the same risk of being affected by LLINs and the $\pi_{\mathrm{i}}$ value does not change with LLIN deployment. However, under the assumption of fully determined behaviour, the LLINs killed only (a proportion of) those mosquitoes that searched during times when LLIN users were under their nets, thus the $\pi_{i}$ value is brought down merely by selective killing of indoor nocturnal host-searching mosquitoes. With a pre-intervention $\pi_{i}$ value of 0.6 , the minimum annually smoothed $\pi_{\mathrm{i}}$ value varied between 0.39 (for the susceptible 'Zeneti' population) and 0.58 (for the resistant 'Yaokoffikro' population), depending on the mosquito population (Figure 2). This range includes those values observed in areas having high coverage of bed nets $[8,16,30,31]$. Assuming some degree of determinedness in host-searching behaviour, the pre-intervention $\pi_{\mathrm{i}}$ value (in the absence of LLINs) in these areas was likely higher [32], and a pre-intervention $\pi_{i}$ value of 0.6 seems a reasonable lower limit to work with for primary malaria vectors.

In these simulations, the periodic emergence rate of new adult mosquitoes was independent of the number of adult mosquitoes [26]. This is under the assumptions that mosquito emergence is strongly dependent on breeding site capacity and that survival from egg to emerging adult is strongly regulated by density dependent processes. 


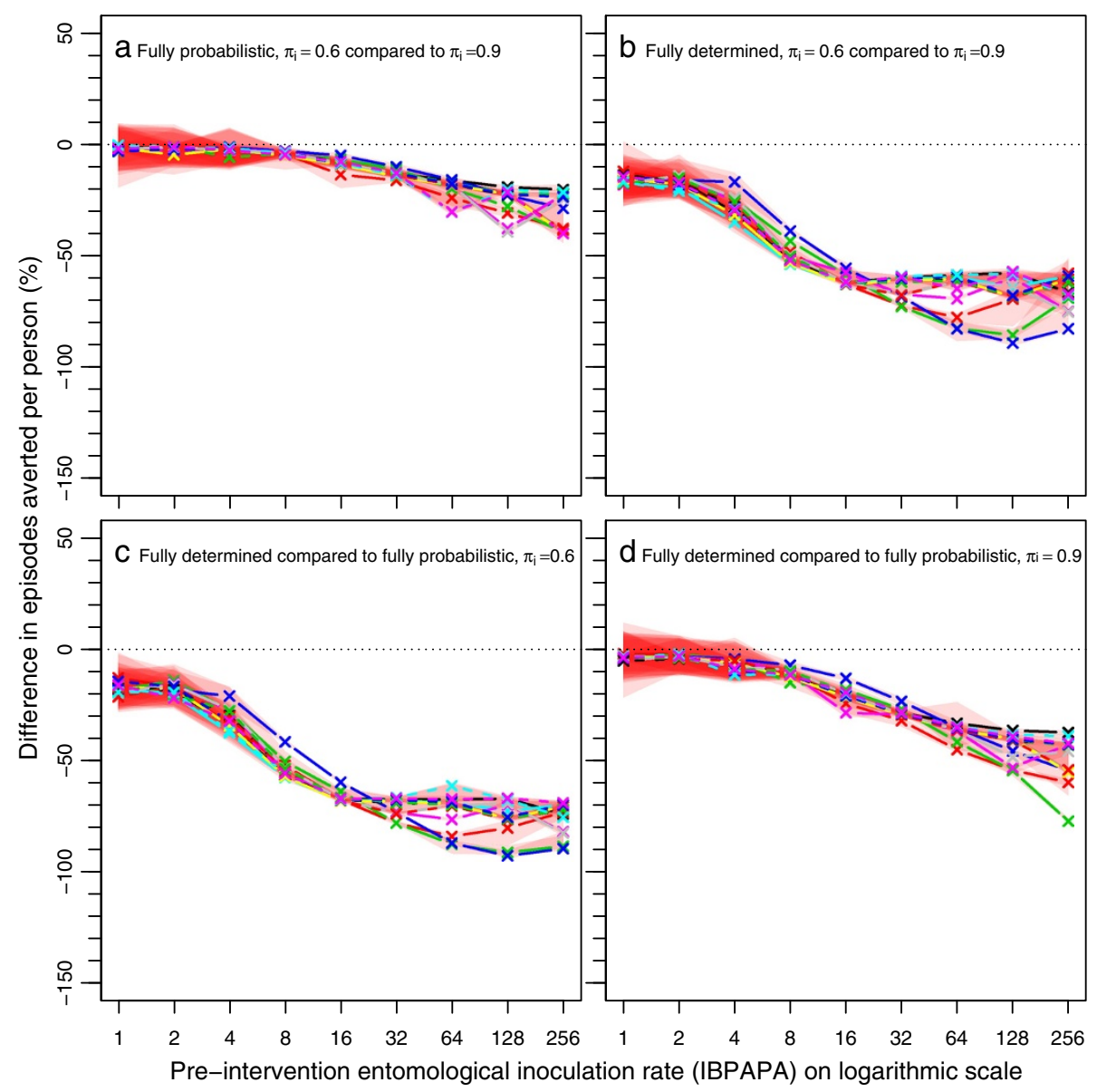

Figure 5 Per cent differences in episodes averted by a mass LLIN distribution, depending on host-searching behaviour. The per cent difference was calculated as $100 \times(A-B) / B$, where $A$ and $B$ are host-searching behaviour assumptions. a) Fully probabilistic host-searching behaviour, with $A$ is behaviour with a $\pi_{i}$ value of 0.6 and $B$ is behaviour with a $\pi_{i}$ value of 0.9 . b) Fully determined host-searching behaviour, with A is behaviour with a $\pi_{i}$ value of 0.6 and $B$ is behaviour with a $\pi_{i}$ value of 0.9 . c) Host-searching behaviour with a $\pi_{i}$ value of 0.6 , with $A$ fully determined behaviour and B fully probabilistic behaviour. $\mathbf{d}$ ) Host searching behaviour with a $\pi_{i}$ value of 0.9 , with $A$ fully determined behaviour and B fully probabilistic behaviour. Horizontal dotted lines are at zero difference in episodes averted. Legend further as in Figure 3.

White and colleagues [33] show that at 50 to $80 \%$ LLIN coverage, these assumptions lead to only small underestimates in the effect of LLINs on the adult mosquito population, compared to models where the emergence rate is dependent on the number of adult mosquitoes (but where density dependent mechanisms still play an important role). Under the assumption of fully probabilistic or not genetically but otherwise determined mosquito host-searching behaviour, the assumption that the emergence rate of new adult mosquitoes is independent of the number of adult mosquitoes has no further consequences. However, if this behaviour is genetically determined (hereditary), higher survival of 'behaviourally resistant' mosquitoes (that bite during times that LLIN users are not under their nets) would lead to relatively more eggs being deposited with alleles associated with this trait (even under the assumption of random mating). Assuming no genetic disadvantage in the larval stage, this would lead to a larger proportion of 'behaviourally resistant' mosquitoes emerging and probably much lower $\pi_{\mathrm{i}}$ values than those observed in areas having high coverage of bed nets [8,16,30,31]. With continued selection pressure, alleles for crepuscular host-searching behaviour could rapidly spread through the mosquito population and eliminate the nocturnal mosquitoes unless the competition between them is weak [34], and the loss in LLIN cost effectiveness could be much higher than estimated by these models. However, such low $\pi_{\mathrm{i}}$ values have not (yet) been reported.

\section{Conclusions}

The models were very sensitive to assumptions about the degree of the determinedness of mosquito host-searching behaviour when there was heterogeneity in this behaviour. This is in line with results from Molineaux and colleagues [13]. Under the assumption of fully probabilistic mosquito host-searching behaviour, the effectiveness of the LLINs 


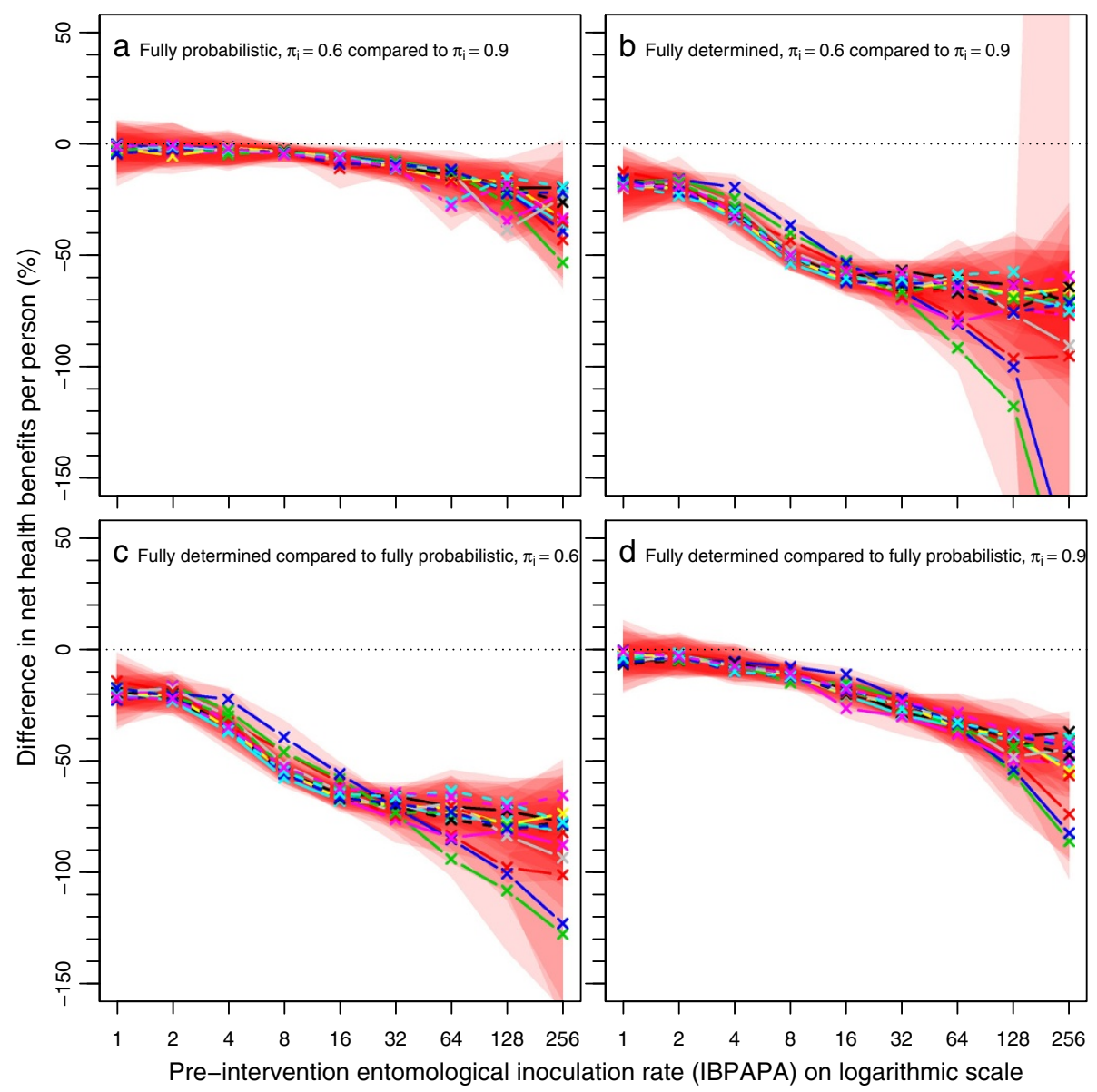

Figure 6 Per cent differences in net health benefits averted by a mass LLIN distribution, depending on host-searching behaviour. Legend as in Figure 5.

appears relatively insensitive to the pre-intervention $\pi_{\mathrm{i}}$ values considered here $\mathrm{e}^{\mathrm{b}}$. Govella and colleagues [16] also use this assumption in a modelling study on the effect of the $\pi_{i}$ value on the efficacy of ITNs. They, too, predict only limited effects, even with pre-intervention $\pi_{\mathrm{i}}$ values as low as 0.46 . However, under the assumption of fully determined mosquito host-searching behaviour, LLIN effectiveness is much more sensitive to the pre-intervention $\pi_{\mathrm{i}}$ value. Although little is known about the degree of determinedness in mosquito host-searching behaviour, evidence that allele frequencies can be associated with outdoorbiting behaviour [11], and lower $\pi_{\mathrm{i}}$ values after exposure to ITNs or LLINs [8] suggests that it is not fully probabilistic. Estimation of LLIN effectiveness should therefore account for the likely non-uniform exposure of vectors [13] and more research on the degree of determinedness of hostsearching behaviour in vector populations is warranted.

The sensitivity analysis (Figure 7) showed that shifts towards crepuscular host-searching behaviour, if this behaviour is determined, can reduce LLIN effectiveness as much as insecticide resistance in nocturnally biting mosquitoes can. However, the effect of the pre-intervention transmission level appears still more important than the effects of physiological and behavioural resistance on the effectiveness of LLINs. The models predicted that a single distribution of LLINs remains cost effective in the simulated scenarios, except for in transmission settings with a pre-intervention inoculation rate of over 64 annual infective bites and physiologically resistant mosquitoes $(70 \%$ mortality in $0.05 \%$ deltamethrin WHO susceptibility tests) that displayed a high proportion (over 40\%) of determined crepuscular host-searching behaviour, where some model variants showed negative NHB (Figure 4c). This is in line with results from a study during a period of increasing bed net use in São Tomé that suggests that bed nets reduced malaria prevalence [35], despite the vector being predominantly early and outdoor biting [36]. However, it should be noted that in most malaria-endemic countries, malaria control will not be limited to a single mass distribution of LLINs and cost-effectiveness may decrease with prolonged LLIN use. 


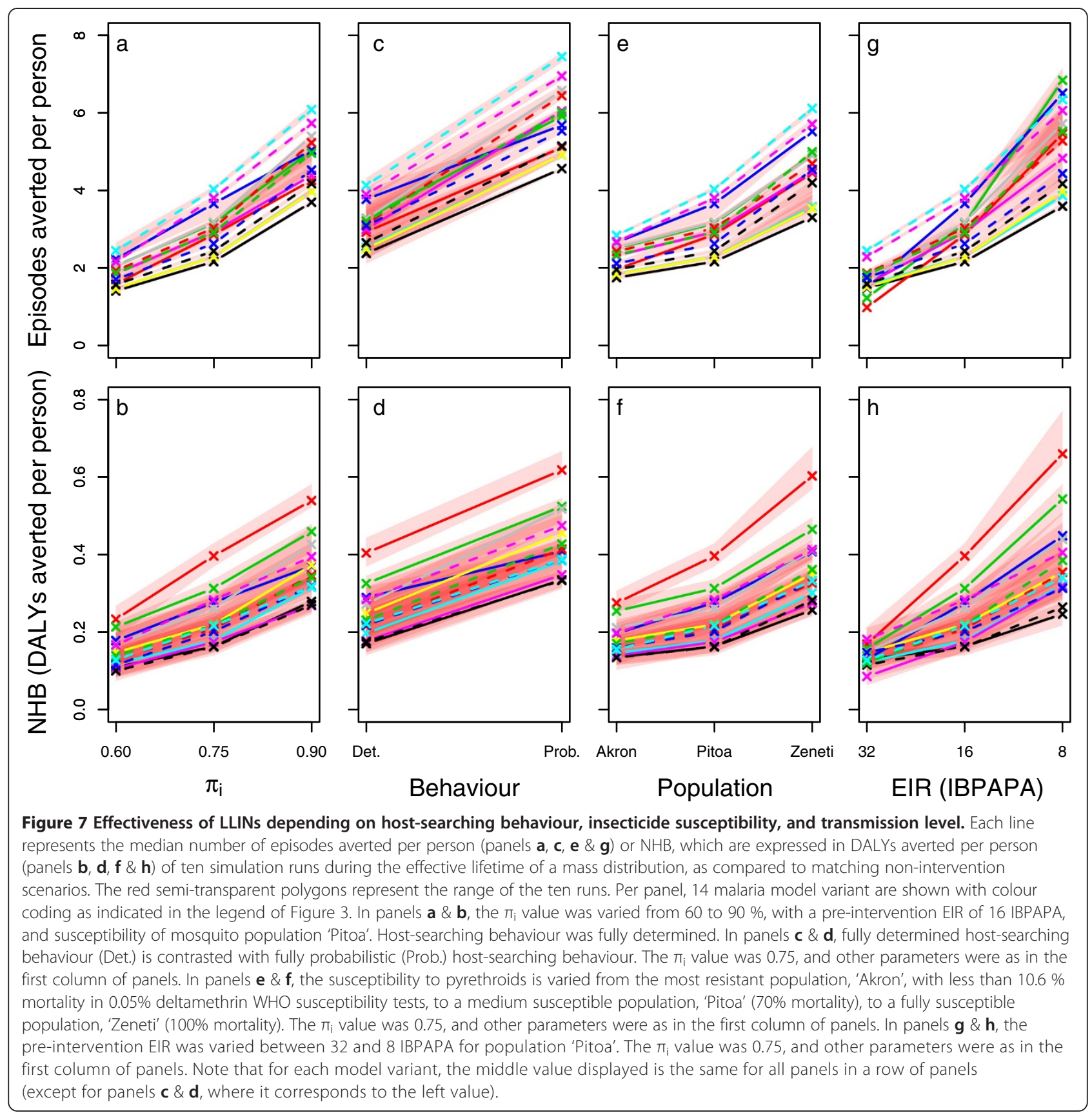

Strong insecticide resistance and strong behavioural resistance against the same vector control intervention are unlikely to occur within the same population. Simulation results in Figure 2 illustrate that in the more insecticideresistant populations (e.g., population 'Akron'), nocturnally host-searching mosquitoes are less suppressed than in insecticide-susceptible populations (e.g., populations 'Zeneti' and 'Malanville'). In this context, it is interesting that the sensitivity of LLIN effectiveness to host-searching behaviour (the $\pi_{i}$ value) is weaker in populations that show insecticide resistance in WHO susceptibility tests as compared to fully susceptible populations [Additional file 2: Figure S3]. The two resistance types likely interfere with each other's development: mosquitoes that are good at avoiding contact with insecticide do not need to develop physiological resistance and mosquitoes that are physiologically resistant do not need to avoid contact with the insecticide. Thus, on the one hand, survival of physiologically susceptible mosquitoes under insecticide pressure has been attributed to avoidance [37-39]. On the other hand, recently documented shifts ${ }^{c}$ in biting rhythm occurred in susceptible populations $[8,40,41]$. Even though the modelling 
results suggest that, for physiologically resistant mosquitoes with $30 \%$ survival in WHO susceptibility tests (population 'Pitoa') that also display determined crepuscular host-searching behaviour, LLINs might not be cost effective in very high transmission settings, evidence for the existence of such 'double resistant' malaria vector populations has yet to be found.

Although models predicted that the LLIN mass distribution would (still) be cost effective against malaria in areas with strong physiological resistance against pyrethroids or where a large proportion of host-mosquito contact occurs during times when LLIN users are not under their nets, in such situations, national malaria control programmes may consider complementing LLINs with other vector control interventions, such as those that are effective against crepuscular and exophagic mosquitoes in order to address the 'residual' transmission [6]. At higher transmission reduction targets than those obtainable with LLINs and case management, such combinations may be more cost effective.

\section{Endnotes}

${ }^{a}$ These experimental huts studies measure effects of LLINs against indoor and nocturnally biting mosquitoes in terms of insecticidal effect and protection against feeding. These effects vary with mosquito population depending on physiological resistance in the population and the degree that mosquitoes from the population are deterred from entering the hut or dissuaded (repelled) from attacking once inside a hut with an LLIN, compared to a control hut. These last two types of mosquito behaviour, resulting in avoidance of contact with the insecticide, are also a form of behavioural resistance against being killed while host searching, just like shifts towards crepuscular host searching behaviour. Nocturnal avoidance of contact with insecticide treated material in otherwise physiologically susceptible mosquitoes [37] could be an important threat to the cost effectiveness of LLINs. Nevertheless, the population level protection against feeding and the population level insecticidal effect in the populations used for parameterization is highly correlated with mortality in WHO susceptibility assays [17], which only measure physiological or biochemical resistance.

${ }^{b}$ With $\pi_{\mathrm{i}}$ values closer to zero, not considered here, sensitivity would increase.

${ }^{\mathrm{c}}$ Although lacking sufficient baseline data to document a shift, a study [42] in Punta Europa on Bioko Island, Equatorial Guinea found unusual high crepuscular and outdoor biting of An. gambiae sensu stricto after several years of indoor residual spraying with pyrethroids followed by several years of spraying with a carbamate insecticide. Although a high frequency of resistance against pyrethroids was found [43], this was not true for resistance against carbamates.

\section{Additional files}

Additional file 1: Figures S1-S2. Differences in episodes and net health benefits averted by a mass LLIN distribution, depending on host-searching behaviour.

Additional file 2: Figure S3. Effectiveness of LLINs depending on host searching behaviour and insecticide susceptibility.

Competing interests

The authors declare that they have no competing interests.

\section{Authors' contributions}

OJTB designed the experiments, analysed results and drafted the manuscript. NC helped conceive of the study and participated in the design. Both authors read and approved the final manuscript.

\section{Acknowledgements}

We acknowledge our colleagues in the Swiss TPH Health Systems Research and Dynamical Modelling Unit, particularly Michael Tarantino, for help with the models and simulations, and thank Amena Briët for help with language editing. Simulations were run with the help of volunteers providing computational power. This work was supported through the Malaria Modelling Project \#OPP1032350 funded by the Bill and Melinda Gates Foundation (BMGF).

Received: 7 March 2013 Accepted: 23 June 2013

Published: 26 June 2013

\section{References}

1. WHO Global Malaria Programme: World Malaria Report 2011. Geneva: World Health Organization; 2011.

2. Hawley WA, Phillips-Howard P, ter Kuile F, Terlouw DJ, Vulule JM, Ombok M, Nahlen B, Gimnig JE, Kariuki SK, Kolczak MS, Hightower AW: Community-wide effects of Permethrin-treated bed nets on child mortality and malaria morbidiy in western Kenya. AmJTrop Med Hyg 2003, 68(Suppl. 4):121-127.

3. Ranson H, N'Guessan R, Lines J, Moiroux N, Nkuni Z, Corbel V: Pyrethroid resistance in African anopheline mosquitoes: what are the implications for malaria control? Trends Parasitol 2011, 27:91-98.

4. Trape JF, Tall A, Diagne N, Ndiath O, Ly AB, Faye J, Dieye-Ba F, Roucher C, Bouganali C, Badiane A, Sarr FD, Mazenot C, Touré-Baldé A, Raoult D, Druilhe P, Mercereau-Puijalon O, Rogier C, Sokhna C: Malaria morbidity and pyrethroid resistance after the introduction of insecticide-treated bednets and artemisinin-based combination therapies: a longitudinal study. Lancet Infect Dis 2011, 11:925-932.

5. Takken W: Do insecticide-treated bednets have an effect on malaria vectors? Trop Med Int Health 2002, 7:1022-1030.

6. Durnez L, Coosemans M: Residual transmission of malaria: an old issue for new approaches. In Anopheles Mosquitoes - New Insights into Malaria Vectors. Edited by Manguin S. Rijeka: Intech; 2013.

7. Gatton ML, Chitnis N, Churcher T, Donnelly MJ, Ghani AC, Godfray HC, Gould F, Hastings I, Marshall J, Ranson H, Rowland M, Shaman J, Lindsay SW: The importance of mosquito behavioural adaptations to malaria control in Africa. Evolution 2013, 67:1218-1230.

8. Russell TL, Govella NJ, Azizi S, Drakeley CJ, Kachur SP, Killeen GF: Increased proportions of outdoor feeding among residual malaria vector populations following increased use of insecticide-treated nets in rural Tanzania. Malar J 2011, 10:80.

9. McCall PJ, Kelly DW: Learning and memory in disease vectors. Trends Parasitol 2002, 18:429-433.

10. Chilaka N, Perkins E, Tripet F: Visual and olfactory associative learning in the malaria vector Anopheles gambiae sensu stricto. Malar J 2012, 11:27.

11. Smits A, Roelants P, Van Bortel W, Coosemans M: Enzyme polymorphisms in the Anopheles gambiae (Diptera: Culicidae) complex related to feeding and resting behavior in the Imbo Valley, Burundi. J Med Entomol 1996, 33:545-553.

12. Bugoro H, Cooper RD, Butafa C, Iro'ofa C, Mackenzie DO, Chen CC, Russell TL: Bionomics of the malaria vector Anopheles farauti in Temotu Province. Solomon Islands: issues for malaria elimination. Malar J 2011, 10:133.

13. Molineaux L, Shidrawi GR, Clarke JL, Boulzaguet JR, Ashkar TS: Assessment of insecticidal impact on the malaria mosquito's vectorial capacity, from 
data on the man-biting rate and age-composition. Bull World Health Organ 1979, 57:265-274

14. Killeen GF, Kihonda J, Lyimo E, Oketch FR, Kotas ME, Mathenge E, Schellenberg JA, Lengeler C, Smith TA, Drakeley CJ: Quantifying behavioural interactions between humans and mosquitoes: evaluating the protective efficacy of insecticidal nets against malaria transmission in rural Tanzania. BMC Infect Dis 2006, 6:161.

15. Seyoum A, Sikaala CH, Chanda J, Chinula D, Ntamatungiro AJ, Hawela M, Miller JM, Russell TL, Briët OJT, Killeen GF: Human exposure to anopheline mosquitoes occurs primarily indoors, even for users of insecticide-treated nets in Luangwa Valley, South-east Zambia. Parasit Vectors 2012, 5:101-105.

16. Govella NJ, Okumu FO, Killeen GF: Insecticide-treated nets can reduce malaria transmission by mosquitoes which feed outdoors. AmJTrop Med Hyg 2010, 82:415-419.

17. Briët OJ, Penny MA, Hardy D, Awolola TS, Van Bortel W, Corbel V, Dabiré RK, Etang J, Koudou BG, Tungu PK, Chitnis N: Effects of pyrethroid resistance on the cost effectiveness of a mass distribution of long-lasting insecticidal nets: a modelling study. Malar J 2013, 12:77

18. Corbel V, Chabi J, Dabiré RK, Etang J, Nwane P, Pigeon O, Akogbeto M Hougard JM: Field efficacy of a new mosaic long-lasting mosquito net (PermaNet ${ }^{\oplus}$ 3.0) against pyrethroid-resistant malaria vectors: a multi centre study in Western and Central Africa. Malar J 2010, 9:113.

19. N'Guessan R, Asidi A, Boko P, Odjo A, Akogbeto M, Pigeon O, Rowland M: An experimental hut evaluation of PermaNet $^{\oplus}$ 3.0, a deltamethrin-piperonyl butoxide combination net, against pyrethroid-resistant Anopheles gambiae and Culex quinquefasciatus mosquitoes in southern Benin. Trans $R$ Soc Trop Med Hyg 2010, 104:758-765.

20. Koudou BG, Koffi AA, Malone D, Hemingway J: Efficacy of PermaNet ${ }^{\oplus} 2.0$ and PermaNet ${ }^{\oplus} 3.0$ against insecticide-resistant Anopheles gambiae in experimental huts in Côte d'Ivoire. Malar J 2011, 10:172

21. Van Bortel W, Chinh VD, Berkvens D, Speybroeck N, Trung HD, Coosemans M: Impact of insecticide-treated nets on wild pyrethroid resistant Anopheles epiroticus population from southern Vietnam tested in experimental huts. Malar J 2009, 8:248.

22. Tungu P, Magesa S, Maxwell C, Malima R, Masue D, Sudi W, Myamba J, Pigeon O, Rowland M: Evaluation of PermaNet 3.0 a deltamethrin-PBO combination net against Anopheles gambiae and pyrethroid resistant Culex quinquefasciatus mosquitoes: an experimental hut trial in Tanzania. Malar J 2010, 9:21.

23. Smith T, Maire N, Ross A, Penny M, Chitnis N, Schapira A, Studer A, Genton B, Lengeler C, Tediosi F, de Savigny D, Tanner M: Towards a comprehensive simulation model of malaria epidemiology and control. Parasitology 2008, 135:1507-1516.

24. OpenMalaria: a simulator of malaria epidemiology and control. [http://code. google.com/p/openmalaria/]

25. Smith T, Killeen GF, Maire N, Ross A, Molineaux L, Tediosi F, Hutton G, Utzinger J, Dietz K, Tanner M: Mathematical modeling of the impact of malaria vaccines on the clinical epidemiology and natural history of Plasmodium falciparum malaria: Overview. AmJTrop Med Hyg 2006, 75(2 Suppl):1-10.

26. Chitnis N, Hardy D, Smith T: A periodically-forced mathematical model for the seasonal dynamics of malaria in mosquitoes. Bull Math Biol 2012, 74:1098-1124.

27. Stinnett AA, Mullahy J: Net health benefits: a new framework for the analysis of uncertainty in cost-effectiveness analysis. Med Decis Making 1998, 18(2 Suppl):S68-S80.

28. Smith T, Ross A, Maire N, Chitnis N, Studer A, Hardy D, Brooks A, Penny M, Tanner M: Ensemble modeling of the likely public health impact of a pre-erythrocytic malaria vaccine. PLOS Med 2012, 9:e1001157.

29. Briët OJT, Hardy D, Smith TA: Importance of factors determining the effective lifetime of a mass, long-lasting, insecticidal net distribution: a sensitivity analysis. Malar J 2012, 11:20.

30. Geissbühler Y, Chaki P, Emidi B, Govella NJ, Shirima R, Mayagaya V, Mtasiwa D, Mshinda H, Fillinger U, Lindsay SW, Kannady K, De Castro MC, Tanner M, Killeen GF: Interdependence of domestic malaria prevention measures and mosquito-human interactions in urban Dar es Salaam. Tanzania. Malar J 2007, 6:126.

31. Russell TL, Lwetoijera DW, Maliti D, Chipwaza B, Kihonda J, Charlwood JD, Smith TA, Lengeler C, Mwanyangala MA, Nathan R, Knols BG, Takken W, Killeen GF: Impact of promoting longer-lasting insecticide treatment of bed nets upon malaria transmission in a rural Tanzanian setting with pre-existing high coverage of untreated nets. Malar J 2010, 9:187.
32. Huho B, Briët O, Seyoum A, Sikaala C, Bayoh N, Gimnig J, Okumu F, Diallo D, Abdulla S, Smith T, Killeen G: Consistently high estimates for the proportion of human exposure to malaria vector populations occurring indoors in rural Africa. Int J Epidemiol 2013, 42:235-247.

33. White MT, Griffin JT, Churcher TS, Ferguson NM, Basanez MG, Ghani AC: Modelling the impact of vector control interventions on Anopheles gambiae population dynamics. Parasit Vectors 2011, 4:153.

34. Murray JD: Models for interacting populations. In Mathematical Biology I: An Introduction. 3rd edition. Edited by Antman SS, Marsden JE, Sirovich L, Wiggins S. New York: Springer; 2002:79-118. Antman SS, Homes P, and Sreenivasan KR (Series Editors): Interdisciplinary Applied Mathematics, vol 17.

35. Charlwood JD, Alcantara J, Pinto J, Sousa CA, Rompao H, Gil V, Rosario VE: Do bednets reduce malaria transmission by exophagic mosquitoes? Trans R Soc Trop Med Hyg 2005, 99:901-904.

36. Charlwood JD, Pinto J, Ferrara PR, Sousa CA, Ferreira C, Gil V, Do Rosario VE: Raised houses reduce mosquito bites. Malar J 2003, 2:45.

37. Kitau J, Oxborough RM, Tungu PK, Matowo J, Malima RC, Magesa SM, Bruce J, Mosha FW, Rowland MW: Species shifts in the Anopheles gambiae complex: Do LLINs successfully control Anopheles arabiensis? PLOS One 2012, 7:e31481.

38. Mathias DK, Ochomo E, Atieli F, Ombok M, Bayoh MN, Olang G, Muhia D, Kamau L, Vulule JM, Hamel MJ, Hawley WA, Walker ED, Gimnig JE: Spatial and temporal variation in the kdr allele L1014S in Anopheles gambiae s.s. and phenotypic variability in susceptibility to insecticides in Western Kenya. Malar J 2011, 10:10

39. Mbogo CN, Baya NM, Ofulla AV, Githure Jl, Snow R: The impact of permethrin-impregnated bednets on malaria vectors of the Kenyan coast. Med Vet Entomol 1996, 10:251-259.

40. Kulkarni MA, Malima R, Mosha FW, Msangi S, Mrema E, Kabula B, Lawrence B, Kinung'hi S, Swilla J, Kisinza W, Rau ME, Miller JE, Schellenberg JA, Maxwell C, Rowland M, Magesa S, Drakeley C: Efficacy of pyrethroid-treated nets against malaria vectors and nuisance-biting mosquitoes in Tanzania in areas with long-term insecticide-treated net use. Trop Med Int Health 2007, 12:1061-1073.

41. Moiroux N, Gomez MB, Pennetier C, Elanga E, Djenontin A, Chandre F, Djegbe I, Guis H, Corbel V: Changes in Anopheles funestus biting behavior following universal coverage of long-lasting insecticidal nets in Benin. J Infect Dis 2012, 206:1622-1629.

42. Reddy MR, Overgaard HJ, Abaga S, Reddy VP, Caccone A, Kiszewski AE, Slotman MA: Outdoor host seeking behaviour of Anopheles gambiae mosquitoes following initiation of malaria vector control on Bioko Island, Equatorial Guinea. Malar J 2011, 10:184-10.

43. Reimer $L$, Tripet F, Slotman M, Spielman A, Fondjo E, Lanzaro GC: An unusual distribution of the kdr gene among populations of Anopheles gambiae on the island of Bioko, Equatorial Guinea. Insect Mol Biol 2005, 14:683-688.

\section{doi:10.1186/1475-2875-12-215}

Cite this article as: Briët and Chitnis: Effects of changing mosquito host searching behaviour on the cost effectiveness of a mass distribution of long-lasting, insecticidal nets: a modelling study. Malaria Journal $201312: 215$

\section{Submit your next manuscript to BioMed Central and take full advantage of:}

- Convenient online submission

- Thorough peer review

- No space constraints or color figure charges

- Immediate publication on acceptance

- Inclusion in PubMed, CAS, Scopus and Google Scholar

- Research which is freely available for redistribution 